\title{
Visual Hallucinations and Cognitive Impairment in Parkinson's Disease
}

\author{
Hee Kyung Park, Jae Seung Kim, Ki Chun Im, Mi Jung Kim, Jae-Hong Lee, \\ Myoung C. Lee, Juhan Kim, Sun Ju Chung
}

\begin{abstract}
Background: Visual hallucination (VH) is a common psychotic symptom in patients with Parkinson's disease (PD) and may be a significant predictor of cognitive impairment (CI) in such patients. Objective: This study aimed to investigate the pattern of glucose metabolism of VH and the relationship between VH and CI in PD. Methods: We studied 28 PD patients, including 15 with VH (PD-VH) and 13 without VH (PD-NVH). Of the 15 PD-VH patients, 8 patients had cognitive impairment (PD-VHCI) whereas 7 did not (PD-VHNCI). All patients underwent $\left[{ }^{18} \mathrm{~F}\right]$ fluorodeoxyglucose positron emission tomography ([ $\left.{ }^{18} \mathrm{~F}\right]$ FDG PET) followed by statistical parametric mapping (SPM) analyses. Results: Compared to the patients with PDNVH, PD-VHNCI patients showed glucose hypometabolism in the inferior and middle temporal cortices, fusiform gyri, and frontal areas, suggesting the involvement of the ventral visual pathway. Compared to the patients with PDNVH, PD-VHCI patients showed glucose hypometabolism in the temporoparietal association cortices with scattered frontal areas. Conclusion: Dysfunction of ventral visual pathway involving the temporal lobe may play a key role in $\mathrm{VH}$ development in PD patients. The evolving distribution from the ventral visual pathway to more extensive posterior cortices in PD-VHCI patients suggests that VH may be a prodromal symptom occurring prior to CI in PD patients.
\end{abstract}

RÉSUMÉ: Hallucinations visuelles et déficit cognitif dans la maladie de Parkinson. Contexte : Les hallucinations visuelles (HV) sont un symptôme psychotique fréquent chez les patients atteints de la maladie de Parkinson (MP) et peuvent constituer un élément de prédiction significatif d'un déficit cognitif (DC) chez ces patients. Objectif : Le but de cette étude était d'examiner le profil du métabolisme du glucose des HV et la relation entre les HV et le DC dans la MP. Méthode : Nous avons étudié 28 patients atteints de MP, dont 15 qui présentaient des HV (MP-HV) et 13 qui n'en avaient pas (MP-NHV). Parmi les 15 patients atteints de MP-HV, 8 patients avaient un DC (MP-HVDC) et 7 n'en avaient pas (MP-HVNDC). Tous les patients ont subi une tomographie par émission de positons au [18F] fluorodéoxyglucose ([18F]FDGPET) et une cartographie statistique paramétrique. Résultats : Les patients atteints de MP-HVNDC avaient un hypométabolisme du glucose dans le cortex temporal inférieur et moyen, la circonvolution occipito-temporale latérale et les zones frontales par rapport aux patients atteints de MP-NHV, ce qui indique que la voie visuelle ventrale pourrait être impliquée. Les patients atteints de MP-HVDC avaient un hypométabolisme du glucose dans les cortex associatifs temporo-pariétaux ainsi que dans des zones frontales dispersées par rapport aux patients atteints de MP-NHV. Conclusion : Une dysfonction de la voie visuelle ventrale impliquant le lobe temporal pourrait jouer un rôle clé dans l'apparition des HV chez les patients atteints de la MP. La distribution évoluant de la voie visuelle ventrale à des zones plus larges du cortex postérieur chez les patients atteints de MP-HVDC indique que les HV pourraient constituer un symptôme avant-coureur d'un DC chez les patients atteints de la MP.

Can J Neurol Sci. 2013; 40: 657-662

Visual hallucination (VH) is the most common psychosis in Parkinson's disease (PD) ${ }^{1,2}$, and is present in $25-50 \%$ of PD patients $\mathrm{s}^{1,3}$. Visual hallucination in PD patients is an important risk factor in terms of nursing home placement, poor quality of life, and mortality ${ }^{4}$. Neuroimaging studies suggested that brain regions involved in higher-order visual processing, ventral or dorsal pathways, were associated with $\mathrm{VH}$ in $\mathrm{PD}$ patients ${ }^{5-9}$. Pathologic study in Lewy body (LB) disease reported a striking association between the distribution of temporal lobe LB and $\mathrm{VH}^{10}$. Visual hallucination in PD seems to be associated with dysfunction of higher-order visual pathways, specifically, the occipitotemporal pathway (the ventral visual pathway). We investigated the regional cerebral glucose metabolism related to $\mathrm{VH}$ in PD patients to identify brain regions, focusing on the ventral visual pathway.

Previous studies excluded patients with cognitive impairment (CI), an important $\mathrm{VH}-$ related nonmotor symptom in $\mathrm{PD}^{8}$, although $\mathrm{VH}$ has been reported to be a possible risk factor for CI development in PD patients ${ }^{11}$. Neuropsychological studies in VH patients without dementia showed reduced verbal fluency ${ }^{12}$ and poor performance in language, verbal learning, and visuoperceptive function ${ }^{13}$. Longitudinal study showed $75 \%$ of

\footnotetext{
From the Department of Neurology (HKP,), Inje University Ilsan-Paik Hospital, Goyang; Department of Neurology (HKP, MJK, JHL, SJC), Department of Nuclear Medicine (JSK, KCI), Asan Medical Center, University of Ulsan College of Medicine; Department of Neurology (HKP, JK), College of Medicine, Hanyang University; Sogang Institute of Advanced Technology (KCI), Sogang University, Seoul; Department of Neurology (MCL), Cheongshim International Medical Center, Gapyeong, Korea.

Received January 10, 2013. Final Revisions Submitted March 11, 2013. Correspondence to: Sun Ju Chung, Department of Neurology, Asan Medical Center University of Ulsan College of Medicine, 88 Olympic-ro 43-gil, Poongnap-dong, Songpa-gu, Seoul, 138-736, Korea. E-mail: sjchung@amc.seoul.kr.
} 
patients with $\mathrm{VH}$ developed dementia ${ }^{14}$. It seems that VH could be a starting point of $\mathrm{CI}$ in PD. We hypothesized that PD patients with $\mathrm{VH}$ and CI may show the identical overlapped areas of metabolic derangement as in PD patients with $\mathrm{VH}$ yet without $\mathrm{CI}$, and still demonstrate less extensive areas of metabolic derangement in PD patients with dementia (PDD). The regions of metabolic derangement in patients with $\mathrm{VH}$ and $\mathrm{CI}$ could provide additional information to predict dementia. Therefore, we also investigated the metabolic alteration related to VH in PD patients with and without CI to shed light on the relationship between $\mathrm{VH}$ and $\mathrm{CI}$.

\section{Materials and Methods}

\section{Subjects}

We prospectively studied 15 PD patients with VH (PD-VH) and 13 PD patients without VH (PD-NVH). Subjects were recruited from the Parkinson/Alzheimer Center of the Asan Medical Center, Seoul, Korea. Parkinson's disease diagnosis was made using the United Kingdom Parkinson's Disease Society Brain Bank criteria ${ }^{15}$. Parkinson's disease-VH was assessed using the neuropsychiatric inventory $(\mathrm{NPI})^{16}$ by a neurologist. We excluded patients with dementia with Lewy bodies (DLB) based on the revised criteria for the clinical diagnosis of DLB ${ }^{17}$. Taking age into consideration, sixteen age-matched normal controls (NC, eight men and eight women, mean age, 64-yearsold) were selected from the data of subjects who visited the Health Promotion Center of Asan Medical Center. Cognitive impairment (CI) was diagnosed on the basis of Korean MiniMental State Examination (K-MMSE) scores; such evaluation considers results from normal Koreans, with regard to age and standard of education ${ }^{18}$. Among the 15 patients in the PD-VH group, 8 had cognitive impairment (PD-VHCI group) whereas 7 did not (PD-VHNCI group). All patients underwent the Montreal Cognitive Assessment-Korean (MoCA-K) ${ }^{19}$ test; these scores were used to correlate clinical data with imaging information. Patients with Parkinson-plus syndromes, secondary parkinsonisms, other neurological diseases, psychiatric disorders, and severe systemic illnesses were all excluded. All included patients provided written informed consent and the Ethics Committee of our institution approved our study protocol.

\section{Clinical assessment}

All patients underwent a comprehensive neurological evaluation; including a detailed neurological examination, routine blood tests, K-MMSE, MoCA-K, Geriatric Depression Scale (GDS), and NPI. Parkinsonian motor features were evaluated using the Unified Parkinson's Disease Rating Scale $(\mathrm{UPDRS})^{20}$ and Hoehn \&Yahr (H\&Y) stage. The axial motor score was defined as the sum of scores on the five UPDRS III subscales: speech, arising from a chair, posture, postural instability, and gait ${ }^{1}$.

\section{$\left[{ }^{18} \mathrm{~F}\right]$ fluorodeoxyglucose positron emission tomography (FDG PET)}

$\left[{ }^{18} \mathrm{~F}\right]$ FDG PET was performed in 15 patients with PD-VH, and 13 patients with PD-NVH. All subjects had fasted for at least six hours. The PET scans were performed in a quiet room using the ECAT HR+ PET scanner (Siemens Medical Systems, Inc.,
Hoffman Estates, IL, USA). A transmission scan of five minutes using a ${ }^{68} \mathrm{Ge}$ rotating pin source and an emission scan of 15 minutes were obtained 40 minutes after $370 \mathrm{MBq}\left[{ }^{18} \mathrm{~F}\right]$ FDG was infused intravenously into each subject. The scanner imaged 63 planes with a slice thickness $2.46 \mathrm{~mm}$ simultaneously for longitudinal field of view of $15.5 \mathrm{~cm}$. All emission images were reconstructed using ordered subset expectation maximization (OSEM), employing 16 subsets and six iterations.

\section{Statistical parametric mapping (SPM) analysis of $\left[{ }^{18}\right.$ F] FDG PET}

$\left[{ }^{18} \mathrm{~F}\right]$ FDG PET images were analyzed using SPM2 (Welcome Functional Imaging Laboratory) in conjunction with MATLAB version 6.5 (The Mathworks, Natick, MA) running on Windows XP. Prior to SPM analysis, all data were normalized using the cerebellum as the area of reference ${ }^{21}$. After conversion of the data from the DICOM file format into the Analyze format, the images from each subject were spatially transformed to a generic SPM2 PET template. Spatially normalized images were smoothed using an isotropic Gaussian Kernel with an 8-mm fullwidth at half-maximum. A two-sided t-test of SPM was performed to assess the group difference. The order of group comparisons was as follows: PD vs. NC, PD-VHNCI vs. NC, PD-VHCI vs. NC, PD-NVH vs. PD-VHNCI, PD-NVH vs. PDVHCI, PD-NVH vs. PD-VH, PD-VHNCI vs. PD-VHCI. The analysis produced a $t$ statistic for each voxel and $t$ values were transformed to standard distribution ( $\mathrm{Z}$ scores). The thresholds chosen in terms of statistical significance were uncorrected $\mathrm{p}<$ 0.01 or uncorrected $\mathrm{p}<0.001$, with the added requirement that at least 50 contiguous voxels exceeded such transformed levels.

To identify brain regions in which glucose metabolism was significantly correlated with the hallucination scores of NPI (NPI-H) in patients with VH, a general linear model using NPI$\mathrm{H}$ as a covariate was tested for each voxel. Positive and negative correlations between glucose metabolism and NPI-H scores were determined. Such analysis was performed on data from PD$\mathrm{VH}$ patients using the covariate-only module of SPM2. To identify brain regions in which cerebral glucose metabolism was significantly correlated with MoCA-K scores in PD-VH patients, a general linear model using the MoCA-K scores as a covariate was tested for each voxel. Positive and negative correlations between glucose metabolism and MoCA-K scores were determined. Confounding variables were included in statistical analyses, including both group analysis and correlation analysis, because of clinical differences between and within groups.

\section{Statistical Analysis}

SPSS for Windows (version 12.0, SPSS Inc., Chicago, IL) was used for statistical analyses and $\mathrm{P}$ values $<0.05$ were regarded as statistically significant. Sex distribution was compared using the Chi-squared test. Differences between clinical groups were analyzed using ANOVA (analysis of variance) combined with the post-hoc Tukey test.

\section{RESULTS}

The clinical characteristics of our patients are described in the Table. There were no differences among the three groups (PD- 
Table 1: Demographic and clinical characteristics of our study subjects

\begin{tabular}{|c|c|c|c|c|}
\hline & PD-VHNCI & PD-VHCI & PD-NVH & $p$ value \\
\hline $\operatorname{Sex}(\mathrm{F}: \mathrm{M})$ & $7(4: 3)$ & $8(3: 5)$ & $13(5: 8)$ & 0.677 \\
\hline Age(years) & $71.0 \pm 4.7$ & $67.8 \pm 6.8$ & $66.3 \pm 5.0$ & 0.210 \\
\hline Education level(year) & $9.1 \pm 3.4$ & $8.3 \pm 5.9$ & $9.2 \pm 5.3$ & 0.915 \\
\hline Disease duration(year) & $5.4 \pm 3.5$ & $6.8 \pm 3.1$ & $5.1 \pm 3.1$ & 0.507 \\
\hline H \& Y stage & $2.0 \pm 0.0$ & $2.2 \pm 0.7$ & $1.5 \pm 0.8$ & 0.077 \\
\hline UPDRS part I-2 score (thought disorder) & $2.3 \pm 0.5^{\ddagger}$ & $2.4 \pm 0.7$ & $0.0 \pm 0.0$ & $<0.001$ \\
\hline UPDRS motor score & $26.8 \pm 13.4$ & $33.9 \pm 11.7$ & $22.1 \pm 12.2$ & 0.148 \\
\hline Axial motor score & $5.3 \pm 2.4^{\ddagger}$ & $4.9 \pm 2.2$ & $2.8 \pm 1.7$ & 0.018 \\
\hline K-MMSE score & $26.1 \pm 1.7$ & $21.6 \pm 5.1$ & $26.9 \pm 1.4$ & 0.002 \\
\hline MoCA-K score & $21.1 \pm 2.3^{\ddagger}$ & $14.0 \pm 3.4$ & $25.6 \pm 2.4$ & $<0.001$ \\
\hline NPI-total score & $19.7 \pm 22.1^{\ddagger}$ & $15.9 \pm 12.9$ & $2.9 \pm 9.1$ & 0.034 \\
\hline NPI hallucination & $5.7 \pm 4.0^{\ddagger}$ & $4.0 \pm 2.6$ & $0.0 \pm 0.0$ & $<0.001$ \\
\hline GDS score & $15.4 \pm 8.1$ & $20.1 \pm 6.9$ & $13.3 \pm 7.7$ & 0.121 \\
\hline Levodopa (mg/day) & $535.7 \pm 223.1$ & $568.8 \pm 329.4$ & $457.7 \pm 178.9$ & 0.563 \\
\hline LED (mg/day) & $537.1 \pm 211.8$ & $616.9 \pm 375.1$ & $531.4 \pm 198.9$ & 0.751 \\
\hline Pramipexole, dose range, mg (No*) & $1.5(1)$ & $0.375-1.5(2)$ & $0.375-1.5(5)$ & \\
\hline Ropinirole, dose range, mg (No.*) & $3-4.5(3)$ & $3-6(4)$ & $0.75-6(8)$ & \\
\hline Selegiline, dose range, $\operatorname{mg}\left(\right.$ No. $\left.^{*}\right)$ & $5(2)$ & $10(1)$ & $5-10(5)$ & \\
\hline Amantadine, dose range, $\mathrm{mg}\left(\right.$ No. $\left.^{*}\right)$ & $100(1)$ & $200(6)$ & $100-200(9)$ & \\
\hline Quetiapine, dose range, mg (No.*) & $12.5-75(5)$ & $25-50(4)$ & & \\
\hline
\end{tabular}

Results are expressed as mean \pm standard deviation. PD-VHNCI, Parkinson's disease patients with visual hallucinations and without cognitive impairment; PD-VHCI, Parkinson's disease patients with visual hallucinations and cognitive impairment; PD-NVH, Parkinson's disease paients without visual hallucinations; F, female; M, male; H\&Y stage, Hoehn and Yahr stage; UPDRS, Unified Parkinson's Disease Rating Scale; Axial motor score, the sum of subscores of UPDRS III subscales (speech, arising from a chair, posture, postural instability, and gait); K-MMSE, Korean-Mini Mental State Examination; MoCA-K, Montreal cognitive assessment-Korean; NPI, neuropsychiatric inventory; GDS, Geriatric depression scale; LED, levodopa equivalent dose; No*, number of treated patients. $\$$ : $\mathrm{p}<0.05$ for comparisons between PD-VHNCI and PD-NVH patients.

VHNCI, PD-VHCI, and PD-NVH) in any of sex distribution, age, educational level, disease duration, $\mathrm{H} \& \mathrm{Y}$ stage, UPDRS part III (motor) scores, GDS, levodopa dose, or levodopa equivalent dose (LED). However, the three groups differed in terms of scores on the UPDRS part I subitem evaluating thought disorder, axial motor subscore, K-MMSE, MoCA-K, NPI-total, and NPI-H. Between PD-NVH and PD-VHNCI groups, there were differences in the scores of UPDRS part I subitem evaluating thought disorder, axial motor subscore, MoCA-K, NPI-total, and NPI-H.
Phenomenology of visual hallucinations consisted of formed hallucinations and minor hallucinations. Formed visual hallucinations consisted of persons (unfamiliar person or family members, or deceased persons), animals, insects, and objects. Minor hallucinations included presence hallucinations and passage hallucinations. Ten patients descried humans and seven patients saw insects. One patient saw a cat and one patient saw a dog. Minor hallucinations occurred in eight patients. A patient with minor hallucinations described that during his exercise, he felt the presenence of another exercising male. Another 


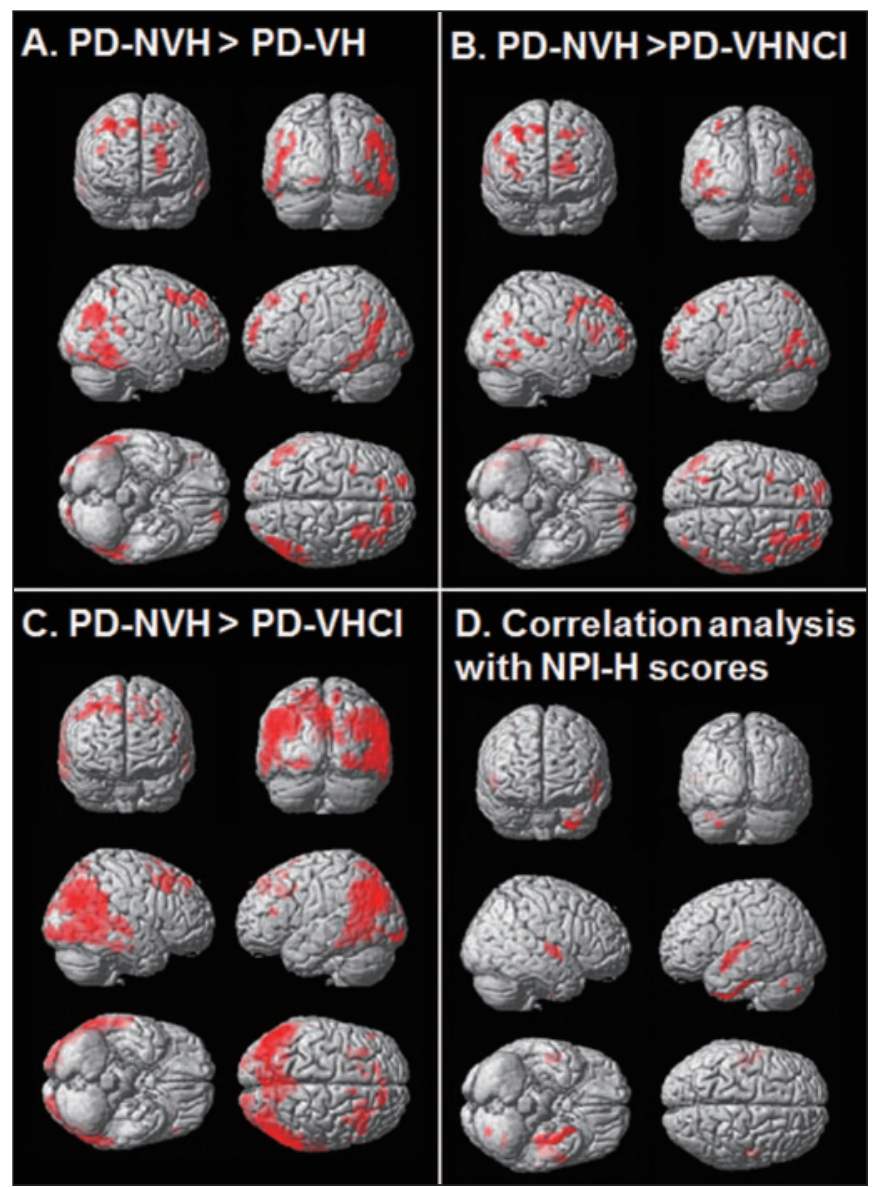

Figure 1: Brain regions associated with VH in PD patients. A. PD-VH group showed glucose hypometabolism in the bilateral middle and inferior temporal cortices, bilateral frontal cortex, left calcarine, left lingual gyrus, and left angular gyrus (uncorrected $p<0.001)$. B. Images from patients with $P D-V H N C I$ and $P D-N V H$, showing that glucose metabolism in the bilateral middle and inferior temporal cortices, bilateral fusiform gyrus, bilateral superior and middle frontal cortices, left superior parietal gyrus, and left middle and inferior occipital cortices of patients with $P D-V H N C I$, was lower than that in patients with $P D$-NVH (uncorrected $p<0.01) C$. PD-VHCI group showed glucose hypometabolism in the bilateral parietotemporal cortices, bilateral frontal cortex, and occipital cortex (uncorrected $p<0.001$ ). D. PD-VH group showed significant negative correlations (uncorrected $p<0.01$ ) between neuropsychiatric inventory-hallucination (NPI-H) scores and the hypometabolism in the superior temporal and fusiform gyri.

description was the transformation of a vacuum cleaner into a person. Five patients experienced auditory hallucinations and visual hallucinations.

\section{Group comparisons using SPM analyses}

There were no significant differences of glucose metabolism between PD patients and normal controls (uncorrected $\mathrm{p}<0.001$ ). Hypometabolic regions in the PD-VH group were bilateral middle and inferior temporal cortices, left lingual gyrus, and left angular gyrus compared with the PD-NVH group (Figure 1A, uncorrected $\mathrm{p}<0.001)$. Glucose metabolism in the bilateral inferior and middle temporal cortices, bilateral frontal areas, and left superior parietal and occipital regions, were lower in the PDVHNCI than in the PD-NVH group (Figure 1B, uncorrected $\mathrm{p}<$ 0.01). SPM analyses between PD-VHCI and PD-NVH groups showed bilateral temporoparietooccipital cortices with lesser involvement of frontal cortex (Figure 1C, uncorrected $\mathrm{p}<0.001$ ). Glucose metabolism in the bilateral precuneus and superior parietal areas, and in scattered regions of the frontal, temporal, and occipital lobes was lower in the PD-VHCI than in the PDVHNCI group (Figure 2A). On all between-group comparisons, the cerebellum was the only region showing an increase in glucose metabolism.

\section{Covariate analysis by SPM analysis}

Covariate analysis showed negative correlations between NPI-H scores and glucose metabolism in the bilateral superior temporal gyri, left fusiform gyrus, and left Heschl's gyrus (Figure 1D). Positive correlations between MoCA-K scores and the extent of glucose metabolism were observed in the bilateral superior parietal and middle occipital gyri, left precuneus, and right fusiform gyrus (Figure 2B).

\section{Discussion}

This study showed that hallucination scores most strongly correlated with the extent of glucose hypometabolism in the superior temporal gyrus and the fusiform gyrus. Furthermore, glucose hypometabolism was observed in the bilateral inferior and middle temporal gyri, and the middle occipital gyrus, in PD$\mathrm{VH}$ and PD-VHNCI groups, suggesting involvement of the

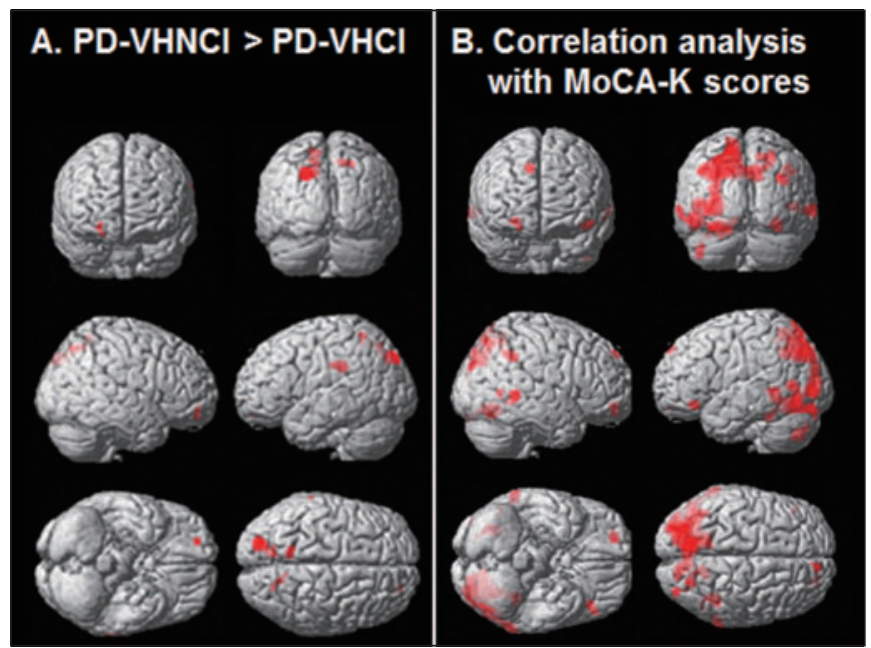

Figure 2: Brain regions associated with cognitive impairment in $P D-V H$ patients. A. Statistical parametric map of the comparisons between $P D$ VHNCI patients and PD-VHCI patients revealed decreased glucose metabolism in the bilateral precuneus and right superior frontal cortex (uncorrected $p<0.001$ ). B. Significant positive correlations (uncorrected $p<0.01)$ between Montreal cognitive assessment-Korean (MoCA-K) scores and the hypometabolism in the bilateral superior parietal and middle occipital gyri, left precuneus, and right fusiform gyrus. 
ventral visual pathway in the $\mathrm{VH}$ in PD patients. Glucose hypometabolism was also observed in the left middle occipital and the superior parietal gyrus of the PD-VH group; the dorsal visual pathway. On the basis of our results, the ventral visual pathway was more affected than the dorsal visual pathway.

The association between temporal lobe and VH in PD patients has been observed in other imaging studies ${ }^{6,22}$ and in pathologic studies $^{10,23}$. One study reported hyperperfusion of the right superior and middle temporal gyri in PD patients with $\mathrm{VH}^{6}$, whereas the other study found that hallucinating PD patients exhibited hypoperfusion in the bilateral inferior parietal lobules and inferior temporal gyrus ${ }^{24}$. Patients having PD with VHs demonstrated increased serotonin $2 \mathrm{~A}$ receptor binding in the ventral visual pathway ${ }^{25}$. Progressive atrophic changes have also been observed in the bilateral superior temporal gyri and frontal areas of PD patients with $\mathrm{VH}^{14}$. Prior pathological studies found an association between $\mathrm{VH}$ and a high density of Lewy bodies in temporal areas ${ }^{10,23,26}$.

Our findings also suggest that $\mathrm{VH}$ may be a prodromal symptom of CI in PD patients. Intraneuronal pathology has been reported to progress upwardly, from the brainstem to the diffuse cortices in PD patients ${ }^{27}$. The temporal cortex is involved at an earlier stage of PD than is the case with other cortices, and the pathologic process extends to other neocortices as PD patients enter the more advanced disease stages of 5 and $6^{27}$. We found that hallucination scores were significantly correlated with glucose hypometabolism in the temporal cortex, and that patients in both PD-VH and PD-VHNCI groups showed glucose hypometabolism in the bilateral inferior and middle temporal gyri. In our PD-VHCI group, the hypometabolic areas extended to diffuse cortical regions, and to a greater extent to posterior cortical regions, compared to frontal regions. Such a pattern of cerebral glucose metabolism suggests that VH may precede CI in PD patients, reflecting the temporal and regional progression of neurodegenerative pathology in PD. Parkinson's disease patients with $\mathrm{VH}$ frequently developed dementia during a mean followup period of 29.9 months and showed extensive brain atrophy, involving gray-matter loss from the limbic, paralimbic, and neocortical areas, as assessed by voxel-based morphometry (VBM) analysis of $\mathrm{MRI}^{14}$; this further suggests that $\mathrm{VH}$ is a prodromal symptom of $\mathrm{CI}$ in PD patients.

In line with previous results, we found that PD-VHNCI group had a higher mean axial motor subscore of $\operatorname{UPDRS}^{3,28}$ and a lower mean MoCA-K score than did our PD-NVH group. Although the K-MMSE scores were similar in our PD-VHNCI and PD-NVH groups, the MoCA-K score was lower in PDVHNCI than in PD-NVH group. These findings suggest that most hallucinating PD patients have mild CI that is not detected by K-MMSE but that may contribute to the development of dementia in PD patients.

In contrast to previous neuroimaging studies, we categorized hallucinating PD patients according to the presence of CI. Thus, we were able to compare cerebral glucose metabolism associated only with $\mathrm{VH}$ with such metabolism associated with both $\mathrm{VH}$ and $\mathrm{CI}$ in PD patients.

Our study had limitations. First, the number of subjects was relatively small. However, our sample size was similar to other functional imaging studies on PD patients. Our data interpretation does not suffer from any obvious limitation due to sample size. Second, extensive neuropsychological tests were not performed. But, we performed MoCA-K that has been reported to be a well-suited screening tool for cognitive impairment in $\mathrm{PD}^{29}$; this screening tool might provide additional information to assess $\mathrm{CI}$ in our PD patients.

In summary, our study provides an additional evidence of impairment of ventral visual pathways in PD patients with $\mathrm{VH}$, highlighting the important role of temporal lobe in $\mathrm{VH}$.

\section{ACKNOWLEDGEMENT}

This study was supported by a grant (2010-0416) from the Asan Institute for Life Sciences, Seoul, Korea. This work was supported by the 2012 Inje University Research Grant.

\section{REFERENCES}

1. Fenelon G, Mahieux F, Huon R, Ziegler M. Hallucinations in Parkinson's disease: prevalence, phenomenology and risk factors. Brain. 2000;123:733-45.

2. Ravina B, Marder K, Fernandez HH, et al. Diagnostic criteria for psychosis in Parkinson's disease: report of an NINDS, NIMH work group. Mov Disord. 2007;22(8):1061-8.

3. Williams DR, Lees AJ. Visual hallucinations in the diagnosis of idiopathic Parkinson's disease: a retrospective autopsy study. Lancet Neurol. 2005;4(10):605-10.

4. Goetz CG, Stebbins GT. Risk factors for nursing home placement in advanced Parkinson's disease. Neurology. 1993;43(11): 2227-9.

5. Ramírez-Ruiz B, Martí MJ, Tolosa E, et al. Cerebral atrophy in Parkinson's disease patients with visual hallucinations. Eur J Neurol. 2007;14(7):750-6.

6. Oishi N, Udaka F, Kameyama M, Sawamoto N, Hashikawa K, Fukuyama H. Regional cerebral blood flow in Parkinson disease with nonpsychotic visual hallucinations. Neurology. 2005;65 (11):1708-15.

7. Matsui H, Nishinaka K, Oda M, et al. Hypoperfusion of the visual pathway in parkinsonian patients with visual hallucinations. Mov Disord. 2006;21(12):2140-4.

8. Boecker H, Ceballos-Baumann AO, Volk D, Conrad B, Forstl H, Haussermann P. Metabolic alterations in patients with Parkinson disease and visual hallucinations. Arch Neurol. 2007 July;64 (7):984-8.

9. Nagano-Saito A, Washimi Y, Arahata Y, et al. Visual hallucination in Parkinson's disease with FDG PET. Mov Disord. 2004;19(7): 801-6.

10. Harding AJ, Broe GA, Halliday GM. Visual hallucinations in Lewy body disease relate to Lewy bodies in the temporal lobe. Brain. 2002;125(2):391-403.

11. Aarsland D, Bronnick K, Ehrt U, et al. Neuropsychiatric symptoms in patients with Parkinson's disease and dementia: frequency, profile and associated care giver stress. J Neurol Neurosurg Psychiatry. 2007;78(1):36-42.

12. Santangelo G, Trojano L, Vitale C, et al. A neuropsychological longitudinal study in Parkinson's patients with and without hallucinations. Mov Disord. 2007;22(16):2418-25.

13. Ramirez-Ruiz B, Junque C, Marti MJ, Valldeoriola F, Tolosa E. Neuropsychological deficits in Parkinson's disease patients with visual hallucinations. Mov Disord. 2006;21(9):1483-7.

14. Ibarretxe-Bilbao N, Ramirez-Ruiz B, Junque C, et al. Differential progression of brain atrophy in Parkinson's disease with and without visual hallucinations. J Neurol Neurosurg Psychiatry. 2010;81(6):650-7.

15. Hughes AJ, Daniel SE, Kilford L, Lees AJ. Accuracy of clinical diagnosis of idiopathic Parkinson's disease: a clinicopathological study of 100 cases. J Neurol Neurosurg Psychiatry. 1992;55(3):181-4.

16. Cummings JL, Mega M, Gray K, Rosenberg-Thompson S, Carusi DA, Gornbein J. The Neuropsychiatric Inventory: comprehensive assessment of psychopathology in dementia. Neurology. 1994;44(12):2308-14. 
17. McKeith IG, Dickson DW, Lowe J, et al. Diagnosis and management of dementia with Lewy bodies: third report of the DLB Consortium. Neurology. 2005;65(12):1863-72.

18. Kang YW. A normative study of the Korean-mini mental state examination (K-MMSE) in the elderly. Korean J Psychol. 2006; 25:1-12.

19. Lee J-Y, Dong Woo Lee, Cho S-J, et al. Brief screening for mild cognitive impairment in elderly outpatient clinic: validation of the Korean version of the Montreal cognitive assessment. J Geriatr Psychiatry Neurol. 2008;21(2):104-10.

20. Fahn S, Elton RL, Members of the UPDRS Development Committee. The Unified Parkinson's Disease Rating Scale. In Fahn S, Marsden CD, Goldstein M, Calne DB, eds: Recent Developments in Parkinson's Disease, Vol. 2. Florham Park, NJ: Macmillan Healthcare Information, 1987. pp. 153-163, 293-304.

21. Soonawala D, Amin T, Ebmeier KP, et al. Statistical parametric mapping of (99m)Tc-HMPAO-SPECT images for the diagnosis of Alzheimer's disease: normalizing to cerebellar tracer uptake. Neuroimage. 2002;17(3):1193-202.

22. Klein RC, de Jong BM, de Vries JJ, Leenders KL. Direct comparison between regional cerebral metabolism in progressive supranuclear palsy and Parkinson's disease. Mov Disord. 2005;20(8):1021-30.
23. Gallagher DA, Parkkinen L, O'Sullivan SS, et al. Testing an aetiological model of visual hallucinations in Parkinson's disease. Brain. 2011;134:3299-309.

24. Matsui H, Nishinaka K, Oda M, et al. Hypoperfusion of the visual pathway in parkinsonian patients with visual hallucinations. Mov Disord. 2006;21(12): 2140-4.

25. Ballanger B, Strafella AP, van Eimeren T, et al. Serotonin 2A receptors and visual hallucinations in Parkinson disease. Arch Neurol. 2010;67(4):416-21.

26. Papapetropoulos S, McCorquodale DS, Gonzalez J, Jean-Gilles L, Mash DC. Cortical and amygdalar Lewy body burden in Parkinson's disease patients with visual hallucinations. Parkinsonism Relat Disord. 2006;12(4):253-6.

27. Braak H, Del Tredici K, Rub U, de Vos RA, Jansen Steur EN, Braak E. Staging of brain pathology related to sporadic Parkinson's disease. Neurobiol Aging. 2003;24(2):197-211.

28. de Maindreville AD, Fenelon G, Mahieux F. Hallucinations in Parkinson's disease: a follow-up study. Mov Disord. 2005;20(2): 212-7

29. Dalrymple-Alford JC, MacAskill MR, Nakas CT, et al. The MoCA: well-suited screen for cognitive impairment in Parkinson disease. Neurology. 2010;75(19):1717-25 